\title{
de Haas-van Alphen Oscillations in a Superconducting State at High Magnetic Fields
}

\author{
Saša Dukan* and Zlatko Tešanović \\ Department of Physics and Astronomy, Johns Hopkins University, Baltimore, MD 21218, USA
}

\begin{abstract}
Low-temperature quantum oscillations of the dHvA amplitude are shown to persist far below the upper critical field of a strongly type-II superconductor, due to the gapless nature of the BCS quasiparticle spectrum in high fields. The dHvA amplitude in the superconducting state is smaller than its normal state counterpart by the factor $\sim[\max (T, \Gamma) / \Delta]^{2}$, where $\Gamma$ is the damping. This factor reflects the presence of a small gapless portion of the Fermi surface, surrounded by regions where the BCS gap is large. The agreement with recent experimental data on $V_{3} S i$ is very good.
\end{abstract}

Typeset using REVTEX

*On leave from Rugjer Bošković Institute, P.O. Box 1016, Zagreb, Croatia 
There has been much interest lately in properties of high $\mathrm{T}_{c}$ superconductors (HTS) and other strongly type-II systems at high magnetic fields [1]. Recent reports clearly demonstrate the de Haas-van Alphen effect in the mixed state of A15 superconductors $\left(V_{3} \mathrm{Si}\right.$ and $\mathrm{Nb}_{3} \mathrm{Sn}$ ), as well as in the layered superconductor $2 \mathrm{H}-\mathrm{NbSe}_{2}$ [2 [4]. In all cases it was found that quantum oscillations in the dHvA amplitude persist to a surprisingly low fraction $(\sim$ $60 \%$ ) of the upper critical field $H_{c 2}$. This is surprising since, in the standard AbrikosovGor'kov theory, one expects an exponential suppression of dHvA amplitude due to the large superconducting gap $\Delta$ at the Fermi surface. Furthermore, it was found that the dHvA amplitude for the fixed value of magnetic field, $H$, behaves as a function of temperature, $T$, in the same way as in the normal state, except for the overall reduction in magnitude when the sample becomes superconducting. The presence of these dHvA oscillations in the mixed phase could, in principle, be due to a small portion of the sample remaining normal. However, heat capacity measurements have ruled out this possibility [2, 3].

In this Letter we show that these experimental results are a direct manifestation of a qualitatively new nature of the BCS quasiparticle spectrum at high fields. As shown in Refs. [5.6], at fields near $H_{c 2}$, this spectrum is gapless at the set of points in the magnetic Brillouin zone (MBZ). These nodes in the gap reflect the center-of-mass motion of Cooper pairs in high magnetic field, in contrast to the familiar nodes of a zero-field unconventional [i.e., por d-wave] anisotropic superconductor which are due to the relative orbital motion. The gapless behavior persists to a relatively low fraction of $H_{c 2}$ [6], as long as $\Delta(T, H)$ remains smaller than or comparable to $\hbar \omega_{c}$, where $\Delta(T, H)$ is the average BCS gap and $\omega_{c}=e H / m c$ is the cyclotron frequency. The results of Corcoran et al. then follow from the presence of a small portion of the Fermi surface containing a coherent gapless band of quasiparticles, while the rest of it is gapped by a large $\Delta$ [7]. High magnetic fields, low temperatures and clean samples provide ideal conditions for the validity of this picture, with the coherent quasiparticle propagation extending over many unit cells of the vortex lattice. For example, the $V_{3} S i$ sample used in experiment [2] satisfies well the last condition, with its electronic mean-free path, $l_{0}$, being much longer than the separation between the vortices (given by the 
magnetic length $l \equiv \sqrt{c / 2 e H}): l_{0}=1450 \AA$ at $T=1.3 \mathrm{~K}$, while the intervortex separation varies from $\sim 60 \AA$ to $\sim 45 \AA$ for fields between 10 and 18.5 Tesla. This situation should be contrasted with the one at low fields, where coherent propagation is suppressed and the low-lying quasiparticle excitations are localized in the cores of isolated vortices.

At high magnetic fields, the electrons participating in superconducting pairing occupy a well-defined set of Landau levels (LLs). At low temperatures and high fields, where $\hbar \omega_{c} \gg$ $T, \tau^{-1}\left(\Gamma_{0}=\hbar / 2 \tau\right.$ is the scattering rate) and $\hbar \omega_{c} \gtrsim \Delta$, numerous 'quantum oscillation' effects arise as consequence of this Landau quantization [8]. In this regime the superconducting order parameter $\Delta(\mathbf{r})$ is well-described by the Abrikosov solution [9] confined to the lowest LL for Cooper pairs [10]. The BCS Hamiltonian is diagonalized in the representation of Ref. [11], where electronic states are labeled by the quasi-momentum $\mathbf{q}[\mathbf{q} \perp \mathbf{H}]$ restricted to the first MBZ, momentum $k_{z}$ parallel to the field and the LL index $n$. Cooper pairs are formed from the electrons with the opposite momenta $\left(\mathbf{q}, k_{z}\right)$ and spins belonging to the same (diagonal pairing) or different LLs (off-diagonal pairing) [0, [10]. Near $H_{c 2}$, where $\Delta \ll \hbar \omega_{c}$, the off-diagonal pairing can be treated as a perturbation and the BCS quasiparticle spectrum is obtained analytically [5],6],10]:

$$
\begin{gathered}
E_{k_{z}, \mathbf{q}, n}= \pm \sqrt{\varepsilon_{n}\left(k_{z}\right)^{2}+\left|\Delta_{n n}(\mathbf{q})\right|^{2}} \\
\varepsilon_{n}\left(k_{z}\right)=\frac{\hbar^{2} k_{z}^{2}}{2 m}+\hbar \omega_{c}(n+1 / 2)-\mu
\end{gathered}
$$

where $\mu$ is the chemical potential. The spectrum consist of $n_{c}$ branches $\left(n_{c}=\operatorname{int}\left[\mu / \hbar \omega_{c}\right]\right.$ is the number of occupied Landau levels) in which the superconducting order parameter $\Delta_{n n}(\mathbf{q})$ goes to zero at points $\mathbf{q}_{\mathbf{j}}$, forming a 'vortex lattice' in the $\mathbf{q}$-space. There is a strong linear dispersion around these points (nodes). $\Delta_{n n}(\mathbf{q})$ for different $n$ in (四) behave similarly in the vicinity of and differ considerably only far away from these nodes. At lower fields, where $\Delta$ becomes comparable to $\hbar \omega_{c}$, the off-diagonal pairing must be included on equal footing. The excitation spectrum is found numerically in Ref. [6] and still exhibits gapless behavior in some of the branches, while the gap starts opening up in the rest. Eventually, at yet lower fields, the LL structure is destroyed by large $\Delta$. [6] The presence of these nodes 
and other regions with a very small gap at the Fermi surface, as $\Delta(T, H)$ increases from zero to $\gtrsim \hbar \omega_{c}$, is the key aspect of our theory.

We now consider the dHvA effect in such gapless high-field superconductor in the low temperature limit. The first step is to determine the oscillatory part of the thermodynamic potential, $\Omega_{o s c}(H, T)$. Initially, we consider a homogeneous 3 -D system [like $V_{3} S i, \mathrm{Nb}_{3} S n$ and other A15 type-II superconductors] and then show how the results change for the layered systems like $\mathrm{NbSe}_{2}$.

The quasiparticle contribution to the thermodynamic potential per unit volume is given by:

$$
\Omega_{\text {exc }}=-\frac{1}{\beta} \sum_{\text {spin }} \frac{1}{L_{x} L_{y} L_{z}} \sum_{n=0}^{n_{c}} \sum_{k_{z} \mathbf{q}}\left\{\ln \left[1+\exp \left(-\beta E_{n}\left(k_{z}, \mathbf{q}\right)\right)\right]+\frac{\beta}{2}\left[E_{n}\left(k_{z}, \mathbf{q}\right)-\varepsilon_{n}\left(k_{z}\right)\right]\right\}
$$

where $\beta=1 / T$ and $E_{n}\left(k_{z}, \mathbf{q}\right)$ is the quasiparticle excitation energy (11). Using the standard Poisson resummation formula:

$$
\sum_{n=0}^{\infty} \phi(n)=\phi(0) / 2+\int_{0}^{\infty} d n \phi(n)+2 R e \sum_{k=1}^{\infty} \int_{0}^{\infty} d n \phi(n) \exp (i 2 \pi k n)
$$

we can perform the sum over Landau level index $n$ in (2) and get the oscillatory part of $\Omega_{\text {exc }}$ as:

$$
\begin{gathered}
\Omega_{o s c}=-\frac{1}{2 \pi^{3} l^{3}} \frac{1}{\beta \hbar \omega_{c}} \sum_{k=1}^{\infty} \frac{(-1)^{k}}{\sqrt{k}} \cos \left(\frac{2 \pi k}{\hbar \omega_{c}} \mu-\frac{\pi}{4}\right) \int_{-\infty}^{\infty} d \varepsilon \exp \left(i 2 \pi k \varepsilon / \hbar \omega_{c}\right) \\
\int_{M B Z} d^{2} \tilde{q}\left\{\ln \left[1+\exp \left(-\beta \sqrt{\varepsilon^{2}+|\Delta(\tilde{\mathbf{q}})|^{2}}\right)\right]+\frac{\beta}{2}\left[\sqrt{\varepsilon^{2}+|\Delta(\tilde{\mathbf{q}})|^{2}}-\varepsilon\right]\right\}
\end{gathered}
$$

where $\tilde{q}=q l$ is the quasi-momentum rescaled by magnetic length.

In deriving (4) we have assumed a spherical Fermi surface. In real systems, with more complicated Fermi surfaces, $\cos \left(2 \pi k \mu / \hbar \omega_{c}-\pi / 4\right)$ is replaced by $\cos \left(\hbar A / \omega_{c}^{*} m^{*}-\pi / 4\right)$, where $A$ is the extremal cross-sectional area of the Fermi surface in the plane $\perp \mathbf{H}$ and $\omega_{c}^{*}=e H / m^{*} c, m^{*}$ being the effective mass associated with the orbit around $\left.A[12]\right)$. We have also neglected the fact that $\Delta$, the overall amplitude of $\Delta_{n n}(\mathbf{q})$, oscillates as a function of $H$, and have assumed that the oscillatory piece of $\Delta$ is much smaller then its 'smooth' part. This is a good approximation if the number of occupied LLs, $n_{c}$, is large $\left[\sqrt{n_{c}} \gg 1\right]$. 
Furthermore, we have neglected different features in $\Delta_{n n}(\mathbf{q})$ as a function of a LL index $n$ since they are pronounced only away from the gapless points [nodes].

While the systems in question are rather clean [3], some impurities and defects are still present and give rise to a small exponential decay of $\mathrm{dHvA}$ amplitude even in the normal state. Therefore, we should include disorder in our calculations. This is accomplished by introducing the density of states per gapless branch of the spectrum (11) in presence of disorder as:

$$
N(\omega) / N_{n}(0)=\frac{1}{\pi} \Im m \sum_{\mathbf{q}, k_{z}} \frac{\tilde{\omega}+\varepsilon\left(k_{z}\right)}{-\tilde{\omega}^{2}+E\left(\mathbf{q}, k_{z}\right)^{2}}
$$

where $\tilde{\omega}=\omega-\Sigma(\tilde{\omega})$ and $N_{n}(0)$ is the normal density of states at the LL. In the clean limit (no disorder) $N(\omega) / N_{n}(0) \approx(\omega / \Delta)^{2}$ for small energies, reflecting the presence of nodes (gapless points) in the quasiparticle spectrum. This strong energy dependence suggests that the effective scattering rate $\tau^{-1}$ in the superconducting state will be modified relative to the normal scattering rate $\tau_{o}^{-1}$. We take the self-energy $\Sigma(\tilde{\omega})$ to be $\Sigma(\tilde{\omega})=-i \Gamma$, where $\Gamma=\hbar / 2 \tau$, which results in $N_{d i s}(\omega) / N_{n}(0) \approx(\Gamma / \Delta)^{2}+(\omega / \Delta)^{2}$. This is a good approximation in the unitary limit (otherwise the scattering rate depends strongly on energy-we have investigated the behavior of the density of states in presence of various forms of static disorder and will present these results elsewhere [13]). In the end, $\Gamma$ has to be determined self-consistently as $\lim _{\omega \rightarrow 0} i \Sigma(\tilde{\omega})$.

It is known that in conventional superconductors, with a finite gap everywhere in the momentum space, the quasiparticle contribution to $\Omega_{\text {osc }}(H, T)$ is exponentially small at low temperatures because of the large value of $\Delta / T$ (unless the field is very close to $H_{c 2}$, within few percent). On the other hand, in the gapless high-field superconductor described above, there are quasiparticle excitations with momenta $\mathbf{q}$ such that $\Delta(\mathbf{q}) \leq T$ which give a large contribution to the thermodynamic potential (4). Therefore, we divide the MBZ in two different regions. The 'gapless' region (which we label $\mathcal{G}$ ), has $\Delta(\tilde{\mathbf{q}}) \cong \Delta \tilde{q}$ for all $\tilde{q} \leq \tilde{q}_{c}$, with $\tilde{q}_{c} \cong C \max (T / \Delta, \Gamma / \Delta)$ being the radius of $\mathcal{G}$ and $C$ a constant of order unity. Outside of $\mathcal{G}$, where $\Delta(\tilde{\mathbf{q}})>\max (T, \Gamma)$ for all $\tilde{q}$, we will aproximate $\Delta(\tilde{\mathbf{q}})$ with the average value of 
the gap function $\Delta=<\Delta(\tilde{\mathbf{q}})>_{a v}$ [14]. This is the 'gapped' region. In setting the boundaries of $\mathcal{G}$ we have allowed the possibility of $T<\Gamma$, which is actually the case in Ref. [2]. There, for example, $T<0.2 \mathrm{meV}$ while the normal scattering rate for $V_{3} S i$ superconductor is $\Gamma_{0}=0.36$ meV in the normal state (we will show that the self-consistent $\Gamma$ actually increases slightly from the normal state value).

Inserting the density of states (5) in (4) and taking the derivative over $H$, we obtain the oscillatory part of the magnetization due to the qasiparticle excitations from the region $\mathcal{G}$ as:

$$
\begin{aligned}
M_{o s c}^{\mathcal{G}}(H, T)= & -\frac{e \hbar}{m c} \frac{1}{\pi l^{3}} \frac{\mu}{\left(\hbar \omega_{c}\right)^{2}}\left[C \max \left(\frac{T}{\Delta}, \frac{\Gamma}{\Delta}\right)\right]^{2} \sum_{k=1}^{\infty} \frac{(-1)^{k}}{\sqrt{k}} \sin \left(\frac{2 \pi k \mu}{\hbar \omega_{c}}-\pi / 4\right) \\
& \times T / \sinh \left(\frac{2 \pi^{2} k T}{\hbar \omega_{c}}\right) \exp \left(-2 \pi k \Gamma / \hbar \omega_{c}\right)+\mathcal{O}\left(\left[\max \left(\frac{T}{\Delta}, \frac{\Gamma}{\Delta}\right)\right]^{4}\right)
\end{aligned}
$$

The amplitude of dHvA oscillations $\mathcal{A}(H, T)$ of magnetization (6) for the first Fourier harmonic $\mathrm{k}=1$ is:

$$
\mathcal{A}^{\mathcal{G}}(H, T) \propto 2\left[C \max \left(\frac{T}{\Delta}, \frac{\Gamma}{\Delta}\right)\right]^{2} H^{-1 / 2} \frac{T}{\sinh \left(2 \pi^{2} T / \hbar \omega_{c}\right)} \exp \left(-2 \pi \Gamma / \hbar \omega_{c}\right)
$$

which is the same contribution as in the normal state reduced by the factor $G=$ $2[C \max (T / \Delta, \Gamma / \Delta)]^{2}$ (for the moment we assume $\Gamma \sim \Gamma_{0}$ ). Factor 2 in (77) comes from the presence of two gapless points in the First MBZ of the spectrum (11). The result ([7) tells us that, in passing from the normal to the superconducting state, there should be a drop in the magnitude of dHvA oscillations, since now there is only a small fraction $G \ll 1$ of the Fermi surface where there are low-energy excitations. The size of $\mathcal{G}$ is determined by both the total number of nodes at the Fermi surface and the areas in different branches where the BCS gap is very small although not necessarily zero. In presence of finite disorder both will broaden into gapless regions [15]. At low temperatures, such that $T<\Gamma$, this broadening is quite small: only $\sim 3 \%$ of Fermi surface in $V_{3} S i$ is still gapless at $T=1.3 \mathrm{~K}$ by the time magnetic field drops from $H=18.5\left(\sim H_{c 2}\right)$ Tesla to $H=10$ Tesla.

The quasiparticle excitations with momenta outside $\mathcal{G}$, where $\Delta>\max (T, \Gamma)$, contribute to the amplitude of dHvA oscillations as: 


$$
\mathcal{A}^{1-\mathcal{G}}(H, T) \propto(1-G) H^{-1 / 2} \frac{\hbar \omega_{c}}{2 \pi^{2}} f\left(\frac{2 \pi \Delta}{\hbar \omega_{c}}\right) \exp \left(-2 \pi \Delta / \hbar \omega_{c}-2 \pi \Gamma / \hbar \omega_{c}\right)
$$

where $f(x)=1$ for $x=0$ and $f(x)=\sqrt{\pi x / 2}$ for $x \gg 1$ and we have assumed $k=1$. This amplitude is much smaller then $\mathcal{A}^{\mathcal{G}}$ : it is already $\sim 20$ times smaller then (7) at $H=16.5$ Tesla in $V_{3} S i$ system and becomes negligible at lower fields.

Figure 1 shows the field dependence of $\ln \left[\mathcal{A}(H, T) \sinh \left(2 \pi^{2} T / \hbar \omega_{c}\right) H^{1 / 2} T^{-1}\right]$ [Dingle plot] for $V_{3} S i$ sample used in the experiment by Corcoran et al. [2], both in normal and superconducting states. The normal state data (straight line) give the normal scattering rate of $\Gamma_{0}=0.36 \mathrm{meV}$. The dashed line in Figure 1 shows the theoretical plot obtained with $\mathcal{A}(H, T)=\mathcal{A}^{\mathcal{G}}(H, T)+\mathcal{A}^{1-\mathcal{G}}(H, T)$, where we have taken the scattering rate $\Gamma$ equal to its normal value $\Gamma_{0}$. We have assumed $\Delta(H)=\Delta(0) \sqrt{1-H / H_{c 2}}$, which is a good approximation for the range of fields used in the experiment. The comparison of this curve and the experimental points suggests that there is a small increase in the scattering rate in the mixed phase. Indeed, the self-consistent calculation of $\Gamma$ gives [15]:

$$
\Gamma(H)=\sqrt{\frac{\Gamma_{0} \Delta(H)}{2}} \quad \text { for } \quad \Gamma / \Delta<1
$$

Using this value of $\Gamma$ in (7) and (8) we obtain the full line in Figure 1. Our theoretical curve is in excellent agreement with the experimental data. We should note here that we use only a single fitting parameter, $C \sim 1.012$, which yields a reasonable value of $\sim 3 \%$ for the size of $\mathcal{G}$. All other quantities are taken from Ref. [3].

Finally, we address the problem of dHvA oscillations in layered superconductors, like $2 \mathrm{H}-\mathrm{NbSe}_{2}$ and HTS $\mathrm{YBa}_{2} \mathrm{Cu}_{3} \mathrm{O}_{7-\delta}$. The layered systems have been extensively studied ever since Graebner and Robbins [4] reported first observation of dHvA oscillations in $2 H-$ $\mathrm{NbSe}_{2}$ at magnetic fields below $H_{c 2}$. In a layered system there is hopping, $t$, between the layers so that the electron dispersion along the field is given by $t \cos \left(k_{z} d\right)$, where $d$ is the interlayer separation. In this case the oscillatory part of the magnetization due to the quasiparticle excitations around gapless points is given by:

$$
M_{\text {osc }}^{\mathcal{G}}(H, T)=-\frac{e \hbar}{m c} \frac{1}{l^{2} d} \frac{\mu}{\left(\hbar \omega_{c}\right)^{3 / 2} t^{1 / 2}}\left[C \max \left(\frac{T}{\Delta}, \frac{\Gamma}{\Delta}\right)\right]^{2} \sum_{k} \frac{(-1)^{k}}{\sqrt{k}} \sin \left(\frac{2 \pi k \mu}{\hbar \omega_{c}}-\pi / 4\right)
$$




$$
\times T / \sinh \left(\frac{2 \pi^{2} k T}{\hbar \omega_{c}}\right) \exp \left(-2 \pi k \Gamma / \hbar \omega_{c}\right)+\mathcal{O}\left(\left[\max \left(\frac{T}{\Delta}, \frac{\Gamma}{\Delta}\right)\right]^{4}\right),
$$

where we have assumed that $t>\hbar \omega_{c}$. Comparing (6) and (10) we see that magnetization in a layered system has the same form as in the homogeneous 3-D system up to the dimensionless factor $(\sqrt{m t} \pi d)^{-1}$. The dHvA oscillations persist deep in the mixed state because of the gapless region $\mathcal{G}$ on the Fermi surface. However, in the layered superconductor such as $2 \mathrm{H}-\mathrm{NbSe}_{2}$ there is a possibility of the charge density wave instability that can modify the shape of the Fermi surface in the certain parts of the MBZ, so that the results of the simple model presented above might not be quantitatively appropriate. 


\section{REFERENCES}

[1] Y. Onuki, I. Umehara, T. Ebihara, N. Nagai and K. Takita, J. Phys. Soc. Japan 61, 692 (1991); G. Kido, K. Komorita, H. Katayama-Yoshida, and T. Takahashi, J. Phys. Chem. Solids 52, 1465 (1991); F. M. Mueller, D. H. Lowndes, Y. K. Chang, A. J. Arko, and R. S. List, Phys. Rev. Lett. 683928 (1992); C. M. Fowler, B. L. Freeman, W. L. Hults, J. C. Lang, J. C. King, F. M. Mueller, and J. L. Smith, Phys. Rev. Lett. 68, 534 (1992); E. G. Haanappel, W. Joss, I. D. Vagner, P. Wyder, K. Trubenbach, H. Mattausch, A. Simon, F. M. Mueller, and S. Askenazy, Physica C 209, 39 (1993).

[2] R. Corcoran, N. Harrison, S. M. Hayden, P. Meeson, M. Springford, and P. J. van der Wel, Phys. Rev. Lett. 72, 701 (1994).

[3] R. Corcoran, N. Harrison, C. J. Haworth, S. M. Hayden, P. Meeson, M. Springford and P. J. van der Wel, preprint (1994).

[4] An early report of quantum oscillations in superconductors is by J. E. Graebner and M. Robbins, Phys. Rev. Lett. 36, 422 (1976).

[5] S. Dukan, A. V. Andreev and Z. Tešanović, Physica C 183, 355 (1991).

[6] S. Dukan and Z. Tešanović, Phys. Rev. B 49, 13017 (1994).

[7] It is important to stress the qualitative difference between this picture and the approximate calculations of U. Brandt, W. Pesch, and L. Tewordt, Z. für Phys. 201, 209 (1967) and K. Maki, Phys. Rev. B 44, 2861 (1991). These approaches do not produce gapless points and consider only the leading corrections to the normal state coming from finite $\Delta$. Such expansions are valid only very close to $H_{c 2}$. In contrast, all the experimental data of Refs. $[2,3]$ come from the region where $\Delta$ is comparable to the cyclotron gap and always much larger than $T$, which makes these approximate treatments difficult to justify.

[8] Z. Tešanović, M. Rasolt, and L. Xing, Phys. Rev. Lett. 63, 2425 (1989); Z. Tešanović, 
M. Rasolt, and L. Xing, Phys. Rev. B 43, 288 (1991); H. Akera, A. H. MacDonald, S. M. Girvin, and M. R. Norman, Phys. Rev. Lett. 67, 2375 (1991); M. J. Stephen, Phys. Rev. B 43, 1212 (1991); A. K. Rajagopal and J. C. Ryan, Phys. Rev. B 44, 10280 (1991); T. Maniv, A. I. Rom, I. D. Vagner and P. Wyder, Phys. Rev. B 46, 8360 (1992); A. H. MacDonald, H. Akera and M. R. Norman, Phys Rev. B 45, 10147 (1992); M. R. Norman, H. Akera, and A. H. MacDonald, Physica C 196, 43 (1992); For review, see M. Rasolt and Z. Tešanović, Rev. Mod. Phys. 64, 709 (1992); A. H. MacDonald, H. Akera and M. R. Norman, Aust. J. Phys. 46, 333 (1993).

[9] A. A. Abrikosov, Zh. Eksp. Teor. Fiz. 32, 1442 (1957).

[10] M. Rasolt and Z. Tešanović, Rev. Mod. Phys. 64, 709 (1992).

[11] Y. A. Bychkov and E. I. Rashba, Zh. Eksp. Teor. Fiz. 85, 1826 (1983).

[12] I. M. Lifshitz and A. M. Kosevich, Zh. Exsp. Teor. Fiz. 29, 730 (1953).

[13] S. Dukan, to be published.

[14] The average gap can be determined by using the method of M. J. Stephen, Phys. Rev. B 45, 5481 (1992).

[15] The theory for the effects of disorder in the present case is similar to the more familiar Abrikosov-Gor'kov theory of disordered unconventional [p- or d-wave] superconductors with nodes in the gap: See A. A. Abrikosov and L. P. Gorkov, Zh. Eksp. Teor. Fiz. 39, 866 (1961) [Sov. Phys.-JETP 12, 1242 (1961)]; P. A. Lee, Phys. Rev. Lett. 71, 1887 (1993) and references therein. 


\section{FIGURES}

FIG. 1. $\ln \left[\mathcal{A} \sinh (X) H^{1 / 2} T^{-1}\right]$ in $V_{3} S i$ as function of $1 / H$, where $X=2 \pi^{2} T / \hbar \omega_{c}$ and $\mathcal{A}=\mathcal{A}^{\mathcal{G}}(H, T)+\mathcal{A}^{1-\mathcal{G}}(H, T)$. Full circles represent the experimental data of Corcoran et al. [2]. The dashed line is a theoretical result obtained from Eqs. $(7,8)$, with $\Gamma$ set to $\Gamma_{0}=0.36$ meV, while the full line uses $\Gamma$ evaluated self-consistently (9). The vertical dashed line indicates the normal-superconductor transition at $H_{c 2}=18.5$ Tesla. We have used $\Delta_{0}=2.6 \mathrm{meV}$ and the effective mass of $1.6 m_{e}$ and $1.7 m_{e}$ in the normal and vortex state respectively. (from Ref. [3].) 